\title{
DERIVATIONS IN PRIME NEAR-RINGS
}

\author{
XUE-KUAN WANG
}

(Communicated by Maurice Auslander)

\begin{abstract}
Let $N$ be a prime near-ring with center $Z$. The purpose of this paper is to study derivations on $N$. We show two main results: (1) Let $N$ be 2-torsion-free, and let $D_{1}$ and $D_{2}$ be derivations on $N$ such that $D_{1} D_{2}$ is also a derivation. Then either $D_{1}$ or $D_{2}$ is zero if and only if $\left[D_{1}(x), D_{2}(y)\right]=0$ for all $x, y \in N$. (2) Let $n$ be an integer $\geq 1, N$ be $n$ !-torsion-free, and $D$ a derivation with $D^{n}(N)=\{0\}$. Then $D(Z)=\{0\}$.
\end{abstract}

Throughout this paper $N$ always stands for a zero-symmetric left near-ring. An additive endomorphism $D$ of $N$ is called a derivation on $N$ if $D(x y)=$ $x D(y)+D(x) y$ for all $x, y \in N$. According to [1], a near-ring $N$ is said to be prime if $x N y=\{0\}$ for $x, y \in N$ implies $x=0$ or $y=0$.

As the addition of a near-ring is not necessarily commutative, the following Proposition 1 has its own significance.

Proposition 1. Let $D$ be an arbitrary additive endomorphism of $N$. Then $D(x y)=x D(y)+D(x) y$ for all $x, y \in N$ if and only if $D(x y)=D(x) y+x D(y)$ for all $x, y \in N$. Therefore $D$ is a derivation if and only if $D(x y)=D(x) y+$ $x D(y)$.

Proof. Suppose $D(x y)=x D(y)+D(x) y$ for all $x, y \in N$. Since $x(y+y)=$ $x y+x y$ and

$$
D(x(y+y))=x D(y+y)+D(x)(y+y)=x D(y)+x D(y)+D(x) y+D(x) y
$$

and

$$
D(x y+x y)=D(x y)+D(x y)=x D(y)+D(x) y+x D(y)+D(x) y,
$$

we get $x D(y)+D(x) y=D(x) y+x D(y)$, so $D(x y)=D(x) y+x D(y)$.

The converse is proved in a similar way.

Lemma 1. Let $D$ be an arbitrary derivation on $N$. Then $N$ satisfies the following partial distributive laws (for $x, y, z, \in N$ ):

(i) $(x D(y)+D(x) y) z=x D(y) z+D(x) y z$;

(ii) $(D(x) y+x D(y)) z=D(x) y z+x D(y) z$.

Proof. (i) was proved in [1], so we only need to prove (ii). From the associative law and Proposition 1 we have

$$
D((x y) z)=D(x y) z+x y D(z)=(D(x) y+x D(y)) z+x y D(z)
$$

Received by the editors July 2, 1991 and, in revised form, September 22, 1992.

1991 Mathematics Subject Classification. Primary 16Y30. 
and

$$
\begin{aligned}
D(x(y z)) & =D(x) y z+x D(y z)=D(x) y z+x(D(y) z+y D(z)) \\
& =D(x) y z+x D(y) z+x y D(z) .
\end{aligned}
$$

Comparing the two expressions we obtain

$$
(D(x) y+x D(y)) z=D(x) y z+x D(y) z .
$$

Now we prove our first main result, which extends a famous theorem on rings of Posner [2] to near-rings.

Theorem 1. Let $N$ be a 2-torsion-free prime near-ring, and let $D_{1}$ and $D_{2}$ be derivations on $N$ such that $D_{1} D_{2}$ is also a derivation. Then the following two conditions are equivalent:

(i) either $D_{1}=0$ or $D_{2}=0$;

(ii) $\left[D_{1}(x), D_{2}(y)\right]=0$ for all $x, y \in N$.

Proof. (i) $\Rightarrow$ (ii) is obvious. We only prove (ii) $\Rightarrow$ (i). Noting that $D_{1} D_{2}$ is a derivation, we have

$$
D_{1} D_{2}(x y)=x D_{1} D_{2}(y)+D_{1} D_{2}(x) y .
$$

On the other hand, $D_{1}$ and $D_{2}$ are both derivations, so

$$
\begin{aligned}
D_{1} D_{2}(x y) & =D_{1}\left(D_{2}(x y)\right)=D_{1}\left(x D_{2}(y)+D_{2}(x) y\right) \\
& =D_{1}\left(x D_{2}(y)\right)+D_{1}\left(D_{2}(x) y\right) \\
& =x D_{1} D_{2}(y)+D_{1}(x) D_{2}(y)+D_{2}(x) D_{1}(y)+D_{1} D_{2}(x) y .
\end{aligned}
$$

The above two expressions for $D_{1} D_{2}(x y)$ yield

$$
D_{1}(x) D_{2}(y)+D_{2}(x) D_{1}(y)=0 \text { for all } x, y \in N .
$$

Replacing $x$ by $x D_{2}(z)$ in (1), by using Proposition 1 and Lemma 1 we have

$$
\begin{aligned}
0 & =D_{1}\left(x D_{2}(z)\right) D_{2}(y)+D_{2}\left(x D_{2}(z)\right) D_{1}(y) \\
& =\left(D_{1}(x) D_{2}(z)+x D_{1} D_{2}(z)\right) D_{2}(y)+\left(x D_{2}^{2}(z)+D_{2}(x) D_{2}(z)\right) D_{1}(y) \\
& =D_{1}(x) D_{2}(z) D_{2}(y)+x D_{1} D_{2}(z) D_{2}(y)+x D_{2}^{2}(z) D_{1}(y)+D_{2}(x) D_{2}(z) D_{1}(y) \\
& =D_{1}(x) D_{2}(z) D_{2}(y)+x\left(D_{1} D_{2}(z) D_{2}(y)+D_{2}^{2}(z) D_{1}(y)\right)+D_{2}(x) D_{2}(z) D_{1}(y) .
\end{aligned}
$$

In this equality

$$
x\left(D_{1} D_{2}(z) D_{2}(y)+D_{2}^{2}(z) D_{1}(y)\right)=0
$$

because the second factor $D_{1} D_{2}(z) D_{2}(y)+D_{2}^{2}(z) D_{1}(y)=0$ by equality (1) with $x$ replaced by $D_{2}(z)$. Thus we get

$$
D_{1}(x) D_{2}(z) D_{2}(y)+D_{2}(x) D_{2}(z) D_{1}(y)=0 \text { for all } x, y, z, \in N .
$$

Replacing $x$ and $y$ by $z$ in (1), respectively, we obtain

$$
D_{2}(z) D_{1}(y)=-D_{1}(z) D_{2}(y)
$$

and

$$
D_{1}(x) D_{2}(z)=-D_{2}(x) D_{1}(z) \text {. }
$$


Since $N$ is a zero-symmetric left near-ring, (2) becomes

$$
\begin{aligned}
0 & =\left(-D_{2}(x) D_{1}(z)\right) D_{2}(y)+D_{2}(x)\left(-D_{1}(z) D_{2}(y)\right) \\
& =D_{2}(x)\left(-D_{1}(z)\right) D_{2}(y)+D_{2}(x)\left(-D_{1}(z) D_{2}(y)\right) \\
& =D_{2}(x)\left[\left(-D_{1}(z)\right) D_{2}(y)-D_{1}(z) D_{2}(y)\right]
\end{aligned}
$$

for all $x, y, z \in N$. If $D_{2} \neq 0$, by [1, Lemma 3] we have

$$
\left(-D_{1}(z)\right) D_{2}(y)-D_{1}(z) D_{2}(y)=0,
$$

that is,

$$
D_{1}(z) D_{2}(y)=\left(-D_{1}(z)\right) D_{2}(y) \text { for all } y, z \in N .
$$

However, by condition (ii) we have

$$
\begin{aligned}
\left(-D_{1}(z)\right) D_{2}(y) & =D_{1}(-z) D_{2}(y)=D_{2}(y) D_{1}(-z) \\
& =D_{2}(y)\left(-D_{1}(z)\right)=-D_{2}(y) D_{1}(z)=-D_{1}(z) D_{2}(y),
\end{aligned}
$$

that is,

$$
\left(-D_{1}(z)\right) D_{2}(y)=-D_{1}(z) D_{2}(y) \text { for all } y, z \in N .
$$

From (3) and (4) we obtain

$$
2 D_{1}(z) D_{2}(y)=0,
$$

or $D_{1}(z) D_{2}(y)=0$ since $N$ is 2-torsion-free. Hence $D_{1}(z) D_{2}(N)=\{0\}$, but $D_{2} \neq 0$ so $D_{1}(z)=0$ for all $z \in N$. Thus $D_{1}=0$.

As a consequence of Theorem 1, we obtain

Corollary 1 [1]. Let $N$ be a 2-torsion-free prime near-ring, and let $D$ be a derivation on $N$ such that $D^{2}=0$. Then $D=0$.

Proof. It is clear that $D^{2}=0$ is a derivation on $N$, and we have

$$
\begin{aligned}
0 & =D^{2}(x y)=D(x D(y)+D(x) y)=D(x D(y))+D(D(x) y) \\
& =x D^{2}(y)+D(x) D(y)+D(x) D(y)+D^{2}(x) y=2 D(x) D(y),
\end{aligned}
$$

so $D(x) D(y)=0$. Therefore $[D(x), D(y)]=0$ for all $x, y \in N$. From Theorem $1, D=0$.

Using equality (1) in the proof of Theorem 1 we can prove the following interesting result.

Proposition 2. Let $N$ be a near-ring and $D_{1}$ and $D_{2}$ be derivations on $N$ such that $D_{1} D_{2}$ is a derivation. Then $D_{2} D_{1}$ is also a derivation.

Proof. Obviously $D_{2} D_{1}$ is an additive endomorphism of $N$. By equality (1) and Proposition 1 we have

$$
\begin{aligned}
D_{2} D_{1}(x y) & =D_{2}\left(D_{1}(x) y+x D_{1}(y)\right)=D_{2}\left(D_{1}(x) y\right)+D_{2}\left(x D_{1}(y)\right) \\
& =D_{2} D_{1}(x) y+\left(D_{1}(x) D_{2}(y)+D_{2}(x) D_{1}(y)\right)+x D_{2} D_{1}(y) \\
& =D_{2} D_{1}(x) y+x D_{2} D_{1}(y)
\end{aligned}
$$

for all $x, y \in N$. Thus $D_{2} D_{1}$ is a derivation by Proposition 1 . 
Corollary 1 leads us naturally to ask a question: let integer $n \geq 2$, and let $N$ be an $n$ !-torsion-free prime near-ring. If $D$ is a derivation on $N$ such that $D^{n}(N)=\{0\}$, can we conclude $D(N)=\{0\}$ ?

The answer is negative even for rings. A simple counterexample due to Chung, Kobayashi, and Luh [3] is as follows: Let $R$ be the ring of $2 \times 2$ matrices over $G F(P)$, where $P$ is a prime integer greater than 3 and $D$ be the inner derivation induced by $\left[\begin{array}{ll}0 & 1 \\ 0 & 0\end{array}\right]$. Then $N$ is 3!-torsion-free and $D^{3}(R)=\{0\}$, but $D(R) \neq\{0\}$.

Nevertheless we will show that in the case of near-ring $D(Z)=\{0\}$ where $Z$ is the center of $N$.

In order to discuss the question we need to extend Leibniz' rule for derivations of rings to near-rings.

Proposition 3. Leibniz' rule holds in near-rings, namely, for any integer $n \geq 2$ and any $x, y \in N$, it holds that

$$
\begin{aligned}
D^{n}(x y)= & D^{n}(x) y+\left(\begin{array}{c}
n \\
1
\end{array}\right) D^{n-1}(x) D(y)+\cdots+\left(\begin{array}{c}
n \\
i
\end{array}\right) D^{n-i}(x) D^{i}(y) \\
& +\cdots+\left(\begin{array}{c}
n \\
n-1
\end{array}\right) D(x) D^{n-1}(y)+x D^{n}(y) .
\end{aligned}
$$

Proof. Using Proposition 1 and elementary facts about centralizers of elements in group, one can easily prove

$$
D(x) y+n x D(y)=n x D(y)+D(x) y .
$$

Further, we can prove

$$
n D(x) y+n x D(y)=n(D(x) y+x D(y)) \quad \text { for all } x, y \in N \text {. }
$$

Next we prove Leibniz' rule by induction on $n$. When $n=2$ we have

$$
\begin{aligned}
D^{2}(x y) & =D(D(x) y+x D(y)) \\
& =D(D(x) y)+D(x D(y)) \\
& =D^{2}(x) y+D(x) D(y)+D(x) D(y)+x D^{2}(y) \\
& =D^{2}(x) y+2 D(x) D(y)+x D^{2}(y) .
\end{aligned}
$$

Assume Leibniz' rule holds for $n-1$. That is, if $N$ is $(n-1)$ !-torsion-free, then

$$
\begin{aligned}
D^{n-1}(x y)= & D^{n-1}(x) y+\cdots+\left(\begin{array}{c}
n-1 \\
i-1
\end{array}\right) D^{n-i}(x) D^{i-1}(y) \\
& +\left(\begin{array}{c}
n-1 \\
i
\end{array}\right) D^{n-i-1}(x) D^{i}(y)+\cdots+x D^{n-1}(y) .
\end{aligned}
$$


Since $n$ !-torsion-freeness implies $(n-1)$ !-torsion-freeness, by $(5)$ we have

$$
\begin{aligned}
& D^{n}(x y)=D\left(D^{n-1}(x y)\right) \\
& =D\left(D^{n-1}(x) y+\cdots+\left(\begin{array}{c}
n-1 \\
i-1
\end{array}\right) D^{n-i}(x) D^{i-1}(y)\right. \\
& \left.+\left(\begin{array}{c}
n-1 \\
i
\end{array}\right) D^{n-i-1}(x) D^{i}(y)+\cdots+x D^{n-1}(y)\right) \\
& =D\left(D^{n-1}(x) y\right)+\cdots+\left(\begin{array}{c}
n-1 \\
i-1
\end{array}\right) D\left(D^{n-i}(x) D^{i-1}(y)\right) \\
& +\left(\begin{array}{c}
n-1 \\
i
\end{array}\right) D\left(D^{n-i-1}(x) D^{i}(y)\right)+\cdots+D\left(x D^{n-1}(y)\right) \\
& =D^{n}(x) y+D^{n-1}(x) D(y) \\
& +\cdots+\left(\begin{array}{c}
n-1 \\
i-1
\end{array}\right)\left(D^{n-i+1}(x) D^{i-1}(y)+D^{n-i}(x) D^{i}(y)\right) \\
& +\left(\begin{array}{c}
n-1 \\
i
\end{array}\right)\left(D^{n-i}(x) D^{i}(y)+D^{n-i-1}(x) D^{i+1}(y)\right) \\
& +\cdots+D(x) D^{n-1}(y)+x D^{n}(y) \\
& =D^{n}(x) y+\cdots+\left(\begin{array}{c}
n-1 \\
i-1
\end{array}\right) D^{n-i+1}(x) D^{i-1}(y)+\left(\begin{array}{c}
n-1 \\
i-1
\end{array}\right) D^{n-i}(x) D^{i}(y) \\
& +\left(\begin{array}{c}
n-1 \\
i
\end{array}\right) D^{n-i}(x) D^{i}(y)+\left(\begin{array}{c}
n-1 \\
i
\end{array}\right) D^{n-i-1}(x) D^{i+1}(y)+\cdots+x D^{n}(y) \\
& =D^{n}(x) y+\cdots+\left(\begin{array}{c}
n-1 \\
i-1
\end{array}\right) D^{n-i}(x) D^{i}(y)+\left(\begin{array}{c}
n-1 \\
i
\end{array}\right) D^{n-i}(x) D^{i}(y) \\
& +\cdots+x D^{n}(y) \\
& =D^{n}(x) y+\cdots+\left[\left(\begin{array}{c}
n-1 \\
i-1
\end{array}\right)+\left(\begin{array}{c}
n-1 \\
i
\end{array}\right)\right] D^{n-i}(x) D^{i}(y) \\
& +\cdots+x D^{n}(y) \\
& =D^{n}(x) y+\cdots+\left(\begin{array}{c}
n \\
i
\end{array}\right) D^{n-i}(x) D^{i}(y)+\cdots x D^{n}(y) .
\end{aligned}
$$

The proof is completed.

Lemma 2. Let $N$ be a near-ring with center $Z$, and let $D$ be a derivation on $N$. Then $D(Z) \subseteq Z$.

Proof. From Proposition 1, for any $z \in Z$ and any $x \in N$ we have

$$
x D(z)+z D(x)=x D(z)+D(x) z=D(x z)=D(z x)=D(z) x+z D(x) .
$$

It follows that $x D(z)=D(z) x$, that is, $D(z) \in Z$.

Lemma 3. Let $n \geq 2$, and let $N$ be an $n$ !-torsion-free near-ring and $D$ be a derivation with $D^{n}(N)=\{0\}$. Then for each $y \in N$, either $D(y)=0$ or there exists $0<k<n$ such that $D^{k}(y)$ is a nonzero divisor of zero.

Proof. Since $n$ !-torsion-freeness implies $(n-1)$ !-torsion-freeness, we may assume that $D^{n-1}(N) \neq\{0\}$, in which case we choose $x_{0}$ such that $D^{n-1}\left(x_{0}\right) \neq 0$. Assume $D(y) \neq 0$. Then there exists $k$ with $0<k<n$ for which $D^{k}(y) \neq 0$ and $D^{k+1}(y)=0$. 
Using Leibniz' rule we obtain

$$
\begin{aligned}
0= & D^{n}\left(x_{0} D^{k-1}(y)\right)=D^{n}\left(x_{0}\right) D^{k-1}(y)+\left(\begin{array}{c}
n \\
1
\end{array}\right) D^{n-1}\left(x_{0}\right) D^{k}(y) \\
& +\left(\begin{array}{c}
n \\
2
\end{array}\right) D^{n-2}\left(x_{0}\right) D^{k+1}(y)+\cdots \\
= & \left(\begin{array}{c}
n \\
1
\end{array}\right) D^{n-1}\left(x_{0}\right) D^{k}(y)=n D^{n-1}\left(x_{0}\right) D^{k}(y) .
\end{aligned}
$$

We get $D^{n-1}\left(x_{0}\right) D^{k}(y)=0$ since $N$ is $n$ !-torsion-free. So $D^{k}(y)$ is a nonzero divisor of zero.

Now we can prove our second main theorem.

Theorem 2. Let $n$ be an integer $\geq 1$ and $N$ be a prime near-ring with center $Z$, and let $N$ be $n$ !-torsion-free and $D$ a derivation with $D^{n}(N)=\{0\}$. Then $D(Z)=\{0\}$.

Proof. If $n=1$, there is nothing to prove. If $n \geq 2$, suppose $D(Z) \neq\{0\}$. We choose $z \in Z$ such that $D(z) \neq 0$. By Lemmas 2 and 3 , there exists a positive integer $k$ such that $D^{k}(z)$ is a nonzero divisor of zero contained in $Z$. On the other hand, $D(z)$ could not be a divisor by [1, Lemma 3]. The contradiction proves that $D(Z)=\{0\}$.

Finally we drop the condition that $N$ is prime to obtain the following

Theorem 3. Let $n$ be a positive integer and $N$ be an $n$ !-torsion-free near-ring with no divisor of zero, then $N$ admits no nonzero derivation $D$ with $D^{n}=0$.

The proof is immediately obtained by Lemma 3 .

\section{ACKNOWLEDGEMENT}

I would like to thank Professor Howard E. Bell for his valuable suggestions and comments, and the referee for useful suggestions.

\section{REFERENCES}

1. H. E. Bell and G. Mason, On derivations in near-ring, Near-Rings and Near-Fields (G. Betsch, ed.), North-Holland, Amsterdam, 1987, pp. 31-35.

2. E. C. Posner, Derivations in prime rings, Proc. Amer. Math. Soc. 8 (1957), 1093-1100.

3. L. O. Chung, Y. Kobayashi, and J. Luh, Remark on nilpotency of derivations, Proc. Japan Acad. Math. Sci. Ser. A 60 (1984), 329-330.

Department of Mathematics, Hubei University, Wuhan 430062, People's Republic of 